EPJ Web of Conferences 113, 04014 (2016)

DOI: 10.1051/epjconf/201611304014

(C) Owned by the authors, published by EDP Sciences, 2016

\title{
Resolution of resonances with the Lorentz integral transform
}

\author{
Winfried Leidemann ${ }^{1,2, a}$ \\ ${ }^{1}$ Dipartimento di Fisica, Università di Trento, I-38123 Trento, Italy \\ ${ }^{2}$ Istituto Nazionale di Fisica Nucleare, TIFPA, I-38123 Trento, Italy
}

\begin{abstract}
The energy resolution of cross sections calculated with the Lorentz integral transform (LIT) method is investigated. It is shown that a crucial role is played by the density of LIT states obtained in the calculation. The situation is illustrated for the simple case of deuteron photodisintegration in unretarded dipole approximation. In a next step the ${ }^{4} \mathrm{He}$ isoscalar monopole resonance is considered. It is pointed out that in a specific energy interval the density of LIT states may depend on the $A$-body basis chosen for the solution of the LIT equation. Using a central NN potential and choosing a proper $A$ body basis set it is shown that the width of the ${ }^{4} \mathrm{He}$ isoscalar monopole resonance can be determined. A value of $180(70) \mathrm{keV}$ is obtained, which is close to the experimental value of $270(50) \mathrm{keV}$.
\end{abstract}

\section{Introduction}

The LIT approach [1] is an ab initio technique that reduces an $A$-body continuum problem to an $A$-body bound-state like problem. The method is usually applied to perturbation induced inclusive breakup reactions of an $A$-body nucleus, where the corresponding cross section can be described in terms of response functions. The LIT represents an integral transform of such a response function and is just given by the norm of a localized function $\tilde{\Psi}$. The latter is determined by the solution of the LIT equation. Finally, the response function, and thus also the cross section, is obtained via the inversion of the transform. Since the LIT method is an approach with a controlled resolution, one knows in a given LIT calculation up to which limit one is able to resolve structures in the reaction cross section. In most of the past LIT applications the relevant structures, e.g. in electron scattering the quasi-elastic peak, were resolved without greater problems. An exception is the recent LIT-EIHH [2] application for the ${ }^{4} \mathrm{He}$ inelastic isoscalar monopole response, where it was realized that the width of the narrow ${ }^{4} \mathrm{He}$ isoscalar monopole resonance could not be resolved due to a too low density of LIT states in the resonance region. The aim of the present work is to describe how this problem can be overcome.

This work is organized as follows. In Sect. 2 the LIT approach is briefly summarized and the term "LIT state" is explained, in addition, the effect of the density of LIT states is illustrated for a simple example (deuteron photodisintegration in unretarded dipole approximation). In Sect. 3 it is discussed that the LIT state density can be increased by choosing a proper basis for the expansion of the localized function $\tilde{\Psi}$. Results with such a basis are presented, in fact they lead to a determination of the width of the ${ }^{4} \mathrm{He}$ inelastic isoscalar monopole resonance [3].

a e-mail: winfried.leidemann@unitn.it 


\section{LIT method}

The inclusive response function $R\left(E_{f}\right)$ induced by an excitation operator $\theta$ is given by

$$
R\left(E_{f}\right)=\int d E_{f}|\langle f|\theta| 0\rangle|^{2} \delta\left(E_{f}-E_{0}-\omega\right),
$$

where $|0\rangle$ and $|f\rangle$ are ground-state and final-state wave functions of an Hamiltonian $H$ of the $A$-body system under consideration, $E_{0}$ and $E_{f}$ are the corresponding eigenenergies, while $\omega=E_{f}-E_{0}$ is the energy transferred to the system. Instead of calculating the response function $R$ via Eq. (1), where however a knowledge of $|f\rangle$ is necessary, one can determine $R$ via the inversion of the LIT $L$, defined as

$$
L\left(\sigma=\sigma_{R}+i \sigma_{I}\right)=\int d E_{f} \frac{R\left(E_{f}\right)}{\left(E_{f}-\sigma_{R}\right)^{2}+\sigma_{I}^{2}},
$$

where $\sigma_{R}$ controls the position and $\sigma_{I}$ the width of the Lorentzian kernel. Because of the adjustable width, the LIT is a transform with a controlled resolution.

The basic LIT equations for an inclusive reaction induced by an excitation operator $\theta$ read

$$
(H-\sigma) \tilde{\Psi}=\theta|0\rangle, \quad L(\sigma)=\langle\tilde{\Psi} \mid \tilde{\Psi}\rangle .
$$

The solution $\tilde{\Psi}$ is localized and can thus be determined via bound-state techniques. In fact, often an expansion of $\tilde{\Psi}$ is made on a complete localized $A$-body basis, where the number of basis states $N$ is increased up to the point that convergence is obtained for the relevant quantities. For a given $N$ one determines the spectrum $H$ for the so defined basis thus finding $N$ eigenstates $\phi_{n}$ with eigenenergies $E_{n}$. The energies $E_{n}$ define the positions of the above mentioned LIT states. Furthermore, the LIT solution assigns to any $E_{n}$ a Lorentzian with strength $S_{n}=\left|\left\langle\phi_{n}|\theta| 0\right\rangle\right|^{2}$ and width $\sigma_{I}$. The LIT is then simply given by a sum of LIT states positioned at $\sigma_{R}=E_{n}$ with strength $S_{n}$ and width $\Gamma=2 \sigma_{I}$ :

$$
L(\sigma)=\sum_{i=1}^{N} \frac{S_{n}}{\left(\sigma_{R}-E_{n}\right)^{2}+\sigma_{I}^{2}} .
$$

The result should resemble a smooth function without that a single LIT state is sticking out. In other words to any value of $\sigma_{R}$ one should have significant contributions from more than one of the $N$ Lorentzians. In case of a rather large $\sigma_{I}$ a few scattered LIT states will be sufficient. However with such a LIT one can only resolve rather broad structures. In order to disentangle finer structure one needs a smaller $\sigma_{I}$, comparable to the size of the structure to resolve, and thus a higher density of LIT states.

As example for the considerations above the case of deuteron photodisintegration in unretarded dipole approximation is discussed in the following. The total cross section is given by

$$
\sigma_{\text {unret }}(\omega)=4 \pi^{2} \alpha \omega R_{\text {unret }}\left(E_{f}=E_{0}+\omega\right),
$$

where $\omega$ denotes the photon energy and $\alpha$ is the fine structure constant. The transition operator $\theta$ for the response function $R_{\text {unret }}$ is the z-component of the dipole operator.

In Fig. 1 the LIT of $R_{\text {unret }}$ is shown [3] (as final state only the ${ }^{3} P_{1}$ NN partial wave is included). One sees for the $\sigma_{I}$ value of $10 \mathrm{MeV}$ that at lower $\sigma_{R}$ a smooth LIT is obtained with only eleven basis functions. At higher $\sigma_{R}$ the LIT result starts to oscillate showing the underlying structure, in fact one sees single Lorentzians sticking out. The oscillations vanish if the number of basis functions $N$, i.e. the LIT state density, is increased. The situation is similar for $\sigma_{I}=2.5 \mathrm{MeV}$, but oscillations already start at rather low $\sigma_{R}$ and still remain even for $N=51$. To obtain also in this case a smoother LIT one needs to increase the LIT state density further. 


\section{$21^{\text {st }}$ International Conference on Few-Body Problems in Physics}

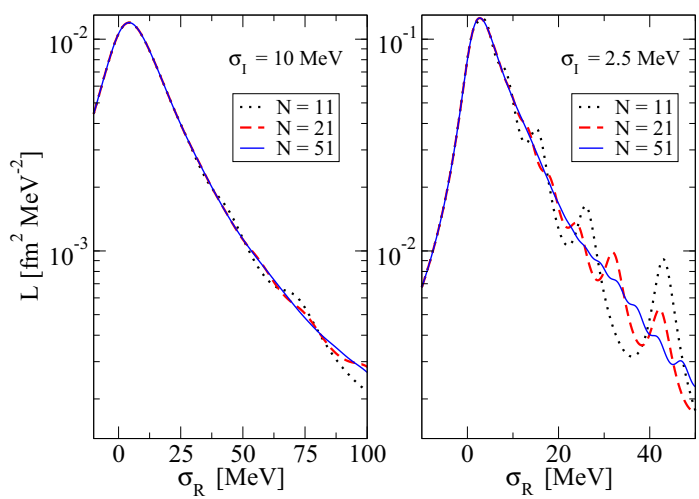

Figure 1. LIT $\mathrm{L}\left(\sigma_{R}, \sigma_{I}\right)$ of the response function $R_{\text {unret }}$ to a specific final state, namely the ${ }^{3} P_{1} \mathrm{NN}$ partial wave. The number of basis functions $N$ used for the expansion of $\tilde{\Psi}$ to solve the LIT equation is as follows: $N=11,21,51$ (calculational details are given in [3]). Left panel: $\sigma_{I}=10 \mathrm{MeV}$. Right panel: $\sigma_{I}=2.5 \mathrm{MeV}$.

\section{LIT approach for narrow resonances}

From the example of the deuteron case above it is evident that the possibility to determine the shape of a narrow cross section structure with the LIT technique is correlated to the density of LIT states. It is important to realize that, in particular at low energy, the density of LIT states depends on the $A$-body basis chosen for the expansion of $\tilde{\Psi}$. In [2] a hyperspherical harmonics (HH) was used and it was found that the LIT state density was not sufficiently high to determine the width of the ${ }^{4} \mathrm{He}$ isoscalar monopole resonance. In fact the resonance is located at a rather low energy, where only a two-body breakup of ${ }^{4} \mathrm{He}$ is possible (bound three-nucleon system plus free fourth nucleon). As a matter of fact an increase of the number of the HH basis states leads mainly to an increase of the LIT state density beyond the three-body breakup threshold, whereas the energy region below is much less affected. In [3] it was shown that it is much better to use a different $A$-body basis such that the dynamical variable of a two-body breakup explicitly appears. For the ${ }^{4} \mathrm{He}$ case considered here it corresponds to the vector pointing from the center-of-mass of the bound three-nucleon system to the position of the fourth nucleon (in Eq. (6) below this variable is called $\mathbf{r}_{4}^{\prime}$ ).

It is worthwhile to mention that in principle the LIT result should not depend on the chosen $A$ body basis. However, this is only the case for a completely converged calculation. Such a complete convergence would imply that the LIT is converged for any value of $\sigma_{I}$ even if the value is extremely small. On the other hand in the limit $\sigma_{I} \rightarrow 0$ one is in principle back to the continuum problem and it is evident that a complete convergence cannot be the aim of a LIT calculation. Nonetheless it is interesting to note that in such a hypothetical fully converged LIT calculation all the necessary information is also encoded in the LIT results with very large $\sigma_{I}$ values. However, the information on a small cross section structure like the shape of narrow resonance would be hidden in tiny contributions. Using such a LIT with large $\sigma_{I}$ would make the inversion unnecessarily difficult. Conversely this means that one should always work with the LIT with the smallest $\sigma_{I}$ that is sufficiently converged. Then one also has a control about the size of structures that can be resolved. In conclusion, in principle in a complete calculation any $A$-body basis leads to the same LIT result, but in reality one has always to work with a limited number of basis states and then it is quite possible that in a given energy interval the density of LIT states depends strongly on the chosen basis.

For the four-body calculation presented here the basis consists of a three-body HH basis with additional two-body short-range correlations ( $\mathrm{CHH}$ basis of [4]), whereas the basis for the fourth nucleon is given by

$$
\left|\Phi\left(\mathbf{r}_{4}^{\prime}\right)\right\rangle=\sum_{l_{4} m_{s_{4}} m_{t_{4}}} \phi_{l_{4}}\left(r_{4}^{\prime}\right)\left|l_{4} m_{l_{4}}\right\rangle\left|s_{4} m_{s_{4}}\right\rangle\left|t_{4} m_{t_{4}}\right\rangle,
$$




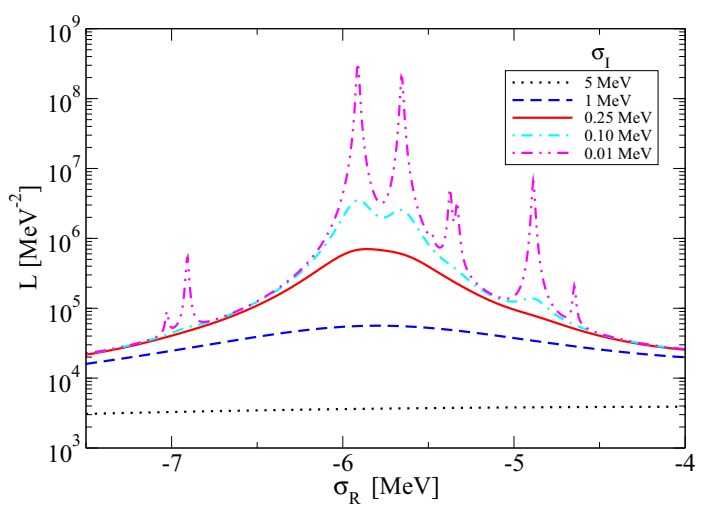

Figure 2. Low-energy part of the LIT L $\left(\sigma_{R}, \sigma_{I}\right)$ for the ${ }^{4} \mathrm{He}$ isoscalar monopole response function with various values of $\sigma_{I}$. The number of basis functions used for the expansion of $\phi_{l_{4}}\left(r_{4}^{\prime}\right)$ is twenty (further details are given in [3]).

where $l_{4}, s_{4}=1 / 2$ and $t_{4}=1 / 2$ are the orbital angular momentum, spin, and isospin quantum numbers, respectively, and $m_{l_{4}}, m_{s_{4}}$ and $m_{t_{4}}$ denote the corresponding projections. The explicit form of the radial wave function $\phi_{l_{4}}\left(r_{4}^{\prime}\right)$ can be found in [3]. The CHH part of the so defined four-body basis provides antisymmetric states for the first three nucleons, whereas the product of the CHH basis states and the single-particle states has to be antisymmetrized in order to have totally antisymmetric basis states for all four particles. The NN potential used in the present study is a central spin dependent NN potential (TN potential) that had been previously employed in the very first LIT applications (electromagnetic break-up of ${ }^{4} \mathrm{He}$ by electrons and real photons $\left.[5,6]\right)$. As discussed in greater detail in [3] the TN potential model leads to a ${ }^{4} \mathrm{He}$ binding energy of $31.41(5) \mathrm{MeV}$.

Concerning the ${ }^{4} \mathrm{He}$ isoscalar monopole resonance one has to consider the isoscalar monopole response function $M(q, \omega)$, where the operator $\theta$ of Eq. (1) becomes $q$-dependent and takes the form

$$
\theta(q)=\frac{G_{E}^{s}\left(q^{2}\right)}{2} \sum_{i=1}^{A} j_{0}\left(q r_{i}\right),
$$

where $G_{E}^{s}\left(q^{2}\right)$ is the nucleon isoscalar electric form factor, $\mathbf{r}_{\mathbf{i}}$ is the position of nucleon $i$, and $j_{0}$ is the spherical Bessel function of $0^{\text {th }}$ order.

In Fig. 2 the low-energy part of the LIT of $M(q, \omega)$ is shown, various $\sigma_{I}$ values are taken. In the resonance region the LIT exhibits a peak which becomes more pronounced for decreasing $\sigma_{I}$. For $\sigma_{I} \geq 0.1 \mathrm{MeV}$ the LIT state density is high enough to lead to a sufficiently smooth LIT, whereas for the smallest $\sigma_{I}$ of $0.01 \mathrm{MeV}$ the single LIT states are clearly sticking out. Via LIT-inversions with $\sigma_{I}=0.1$ and $0.25 \mathrm{MeV}$ the width $\Gamma$ of the resonance can be determined, a value of $\Gamma=180(70) \mathrm{keV}$ is obtained [3], which agrees quite well with the experimental result of 270(50) $\mathrm{keV}$ [7].

\section{References}

[1] V. D. Efros, W. Leidemann, and G. Orlandini, Phys. Lett. B 338, 130 (1994);

V. D. Efros, W. Leidemann, G. Orlandini and N. Barnea, J. Phys. G 34, R459 (2007)

[2] S. Bacca, N. Barnea, W. Leidemann, and G. Orlandini, Phys. Rev. Lett. 110, 042503 (2013)

[3] W. Leidemann, Phys. Rev. C 91, 054001 (2015)

[4] V. D. Efros, W. Leidemann, and G. Orlandini, Phys. Lett. B 408, 1 (1997)

[5] V. D. Efros, W. Leidemann, and G. Orlandini, Phys. Rev. Lett. 78, 432 (1997)

[6] V. D. Efros, W. Leidemann, and G. Orlandini, Phys. Rev. Lett. 78, 4015 (1997)

[7] Th. Walcher, Phys. Lett. B, 31, 442 (1970) 